\title{
Fuzzy Graphs: Algebraic Structure and Syntactic Recognition
}

\author{
Antonios Kalampakas* \\ Department of Production Engineering and Management, \\ Laboratory of Computational Mathematics, \\ Democritus University of Thrace, V.Sofias 12 , \\ Prokat, Building A1, 67100, Xanthi, Greece \\ akalampakas@gmail.com \\ Stefanos Spartalis \\ Department of Production Engineering and Management, \\ Laboratory of Computational Mathematics, \\ Democritus University of Thrace, V.Sofias 12, \\ Prokat, Building A1, 67100, Xanthi, Greece \\ sspart@pme.duth.gr \\ Lazaros Iliadis \\ Department of Forestry \& Management of the Environment \\ \& Natural Resources, Democritus University of Thrace, \\ 193 Pandazidou st., 68200, Nea Orestiada, Greece \\ liliadis@fmenr.duth.gr

\section{Elias Pimenidis} \\ School of Architecture Computing and Engineering, \\ University of East London, 4-6 University Way, London, \\ E16 2RD, United Kingdom \\ e.pimenidis@uel.ac.uk
}

\begin{abstract}
Directed fuzzy hypergraphs are introduced as a generalization of both crisp directed hypergraphs and directed fuzzy graphs. It is proved that the set of all directed fuzzy hypergraphs can be structured into a magmoid with operations graph composition and disjoint union. In this framework a notion of syntactic recognition inside magmoids is defined and several properties are presented.
\end{abstract}

\section{Introduction}

Fuzzy set theory, since its development in 1965, and in general fuzzy structures, have contributed to a wide range of real world applications due to their ability 
*The author has been co-financed by the European Social Fund and Greek national funds through the research funding program: THALIS project "Algebraic Modeling of Topological and Computational Structures and Applications" 
to cover the distance between the numerical models used in engineering and the symbolic models used in formal systems and artificial intelligence [35]. In particular, fuzzy graphs, introduced in 1975, by Rosenfeld are able to model real time systems where the inherent level of information in them varies with different levels of precision [32].

Our intention is to develop a notion of algebraic recognizability for sets of directed fuzzy (hyper)graphs, i.e., directed (hyper)graphs with fuzzy edges and fuzzy nodes, in analogy with the corresponding algebraic recognizability notions for strings [18] and trees [21]. Directed hypergraphs consist of a set of vertices (nodes) and a set of hyperedges, just as ordinary directed graphs except that a hyperedge may have an arbitrary sequence of sources (incoming arrows) and an arbitrary sequence of targets (outgoing arrows), instead of only one source and one target as is the case for ordinary graph edges. Each hyperedge is labeled with a symbol from a doubly ranked alphabet $\Sigma$ in such a way that the first (resp. second) rank of its label equals the number of its sources (resp. targets). Additionally, every hypergraph is equipped with a sequence of begin and end nodes. Ordinary directed graphs are obtained as a special case of directed hypergraphs i.e., in the case that each hyperedge has one source and one target, there are no multiple edges and no edge labels, and the begin and end sequences are the empty word. The fuzzy graphs of [32] are costructed by taking fuzzy subsets of the node and edge sets of a given directed crisp graph.

Engelfriet and Vereijken proved in [19] that every (hyper)graph can be constructed from a finite set of elementary graphs by inductively using the operations of concatenation and sum. Since for every graph an infinite number of such expressions exist, at the same paper, the authors stated the open problem of finding a complete set of equations (rewriting rules) with the property that two expressions represent the same graph if and only if one can be transformed into the other by these equations. This problem was solved in [9] by appropriately adopting magmoids as the necessary algebraic structure for the representation of graphs and led to the construction, for the first time, of automata operating on arbitrary graphs $[10,12,23]$.

A magmoid, introduced by Arnold and Dauchet in 1978, is a doubly ranked set endowed with two operations which are associative, unitary, and compatible to each other $[1,2,8,9,11]$. It generalizes the ordinary monoid structure and a natural regularity notion, analogously with the string case, derives from this. More precisely, we say that a subset $L$ of a magmoid $M$ is recognizable whenever there exist a locally finite magmoid $N$ and a morphism of magmoids $h: M \rightarrow N$, so that $L=h^{-1}(P)$ for some $P \subseteq N$ [11].

The set of directed fuzzy hypergraphs, as defined above, is structured into a magmoid with operations fuzzy graph composition and disjoint union. As a result the previously described recognizability mode can be applied for fuzzy graph languages and the corresponding class is closed under boolean operations, inverse magmoid morphisms and sum operation.

Fuzzy recognizability has been investigated towards various directions, via fuzzy automata [29, 30, 34], syntactic monoids [5, 6], monoid fuzzification [24], nondeterministic recognizability [26], minimization of finite automata [27], infi- 
nite computations [25, 33], and tree recognizability [7, 20].

In the present paper we introduce a syntactic recognizability theory, inside the framework of magmoids, for directed fuzzy hypergraphs analogously with the already established theory for sets of directed crisp graphs [11], see also $[16,17]$. In Section 2 fuzzy graphs are defined by extending the definition of Rosenfeld. Their algebraic structure and several fundamental results are described in the following section. In Section 4 we introduce the recognition mechanism and investigate its properties. A characterization of recognizability is obtained from the underlying magmoid structure which allows us to explore its recognition capacity. Moreover, the closure properties of the corresponding class are examined and several interesting examples are illustrated including the strongly connected fuzzy graphs and the fuzzy complement of a fuzzy graph language.

\section{Fuzzy Graphs}

A fuzzy set $A_{\mu}=(A, \mu)$ consists of a set $A$ together with a function $\mu: A \rightarrow[0,1]$ called the membership function of $A_{\mu}$, for every $x \in A$ the value $\mu(x)$ is called the membership grade of $x$. The support of $A_{\mu}$ is the crisp set

$$
A^{\mu}=\{x \mid x \in A \text { and } \mu(x)>0\} \text {. }
$$

A doubly ranked set, or doubly ranked alphabet, $\left(X_{m, n}\right)_{m, n \in \mathrm{N}}$ is a set $X$ together with a function rank: $X \rightarrow \mathrm{N} \times \mathrm{N}$, where $\mathrm{N}$ is the set of natural numbers. For $m, n \in \mathrm{N}, X_{m, n}=\{x \in A \mid \operatorname{rank}(x)=(m, n)\}$. In what follows we will drop the subscript $m, n \in \mathrm{N}$ and denote a doubly ranked set simply by $X=\left(X_{m, n}\right)$. The set of all words over a finite set $A$ is denoted $A^{*}$, for every word $w \in A^{*}$, $|w|$ denotes its length. We denote by $\varepsilon$ the empty word and $A^{+}=A^{*}-\{\varepsilon\}$. For $n \in \mathrm{N},[n]$ stands for the set $\{1,2, \ldots, n\}$.

A fuzzy $(m, n)$-graph over the doubly ranked alphabet $\Sigma=\left(\Sigma_{m, n}\right)$ is a tuple $G=\left(V_{K}, E_{\lambda}, s, t, l\right.$, begin, end $)$ consisting of

- the nonempty fuzzy set of nodes or vertices $V_{K}=(V, K)$,

- the fuzzy set of edges $E_{\lambda}=(E, \lambda)$, with $E=E_{\lambda}^{\mathbf{s}}$,

- the source and target functions $s: E \rightarrow V^{+}$and $t: E \rightarrow V^{+}$, respectively,

- the labeling function $I: E \rightarrow \Sigma$ such that

$$
\operatorname{rank}(/(e))=(|s(e)|,|t(e)|), \text { for all } e \in E,
$$

- the sequences of begin and end nodes begin $\in V^{*}$ and end $\in V^{*}$ with $\mid$ begin $\mid=$ mand $\mid$ end $\mid=n$.

Notice that vertices can be duplicated in the begin and end sequences of the graph and also at the sources and targets of the edges. For an edge $e$ of a fuzzy graph $G$ we simply write $\operatorname{rank}(e)$ to denote $\operatorname{rank}(/(e))$. This definition is a 
generalization for hypergraphs of the one given by Rosenfeld in [32] (see also [28]). More precisely, if in the above we set

- for every $e \in E, \operatorname{rank}(e)=(1,1)$,

- $\Sigma=\Sigma_{1,1}$ is a singleton set,

- for every pair $(v, u) \in V \times V$ there exists at most one $e \in E$ such that $(s(e), t(e))=(v, u)$,

- for every $e \in E$

$$
\lambda(e) \leq \min \{\kappa(v) \mid v \text { appears in the words } s(e) \text { or } t(e)\},
$$

- and begin $=e n d=\varepsilon$,

then the so obtained constructs are directed unlabeled fuzzy graphs without hyperedges and multiple edges and no begin and end sequences, presented in [32], for simplicity we shall call them binary fuzzy graphs.

The specific sets $V$ and $E$ chosen to define a concrete fuzzy graph $G$ are actually irrelevant. We shall not distinguish between two isomorphic fuzzy graphs. Hence we have the following definition of an abstract graph. Two concrete fuzzy $(m, n)$-graphs

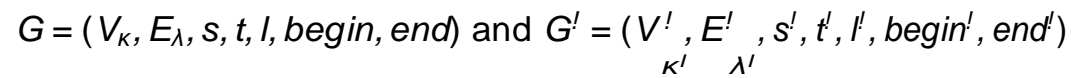

over $\Sigma$ are isomorphic if and only if there exist two bijections $h_{V}: V \rightarrow V !$ and $h_{E}: E \rightarrow E^{!}$commuting with $\kappa, \lambda, s, t$, l, begin and end in the obvious way. An abstract fuzzy $(m, n)$-graph is defined as the equivalence class of a concrete fuzzy $(m, n)$-graph with respect to isomorphism. We denote by $F G R_{m, n}(\Sigma)$ the set of all abstract fuzzy $(m, n)$-graphs over $\Sigma$ and $F G R(\Sigma)=\left(F G R_{m, n}(\Sigma)\right)_{m, n \in \mathrm{N}}$. Since we shall mainly be interested in abstract fuzzy graphs we simply call them fuzzy graphs. A fuzzy graph $G$ is called: discrete if $\lambda(e)=0$ for all $e \in E$, crisp if the sets $V_{k}, E_{\lambda}$ are crisp. Given a binary graph $G=\left(V_{k}, E_{\lambda}, s, t\right.$, l, begin, end $)$, its complement is the binary graph $G^{c}=\left(V_{K}, E_{\lambda^{\prime}}, s, t, l\right.$, begin, end $)$ with

$$
\lambda^{\prime}(e)=\min \{\kappa(s(e)), \kappa(s(e))\}-\lambda(e),
$$

for all $e \in E$. Notice that this definition is accordant with Eq. (1) and moreover it is a generalization of the definition for the complement of a crisp graph. Given a binary fuzzy graph language $L \subseteq F G R(\Sigma)$ its compliment is $L^{c}=\left\{G^{c} \mid G \in\right.$ $L\}$.

Given a fuzzy graph $G=\left(V_{k}, E_{\lambda}, s, t, l\right.$, begin, end $)$ we say that there exists a directed path from the node $v_{1}$ to the node $v_{k}$ of $G$ if there exist edges $e_{1}, \ldots, e_{k-1}$ and nodes $v_{2}, \ldots, v_{k-1}$ of $G$ such that $v_{i}$ appears in $s\left(e_{i}\right)$ and $v_{i+1}$ appears in $t\left(e_{i}\right)$ for all $i=1, \ldots, k-1$. A fuzzy graph $G=\left(V_{k}, E_{\lambda}, s, t, l\right.$, begin, end $)$ is called strongly connected if for all pairs $(u, v) \in V \times V$, with $u j=v$, there exists a directed path from $u$ to $v$. 


\section{Algebraic Structure}

A magmoid (cf. [1, 2, 9, 11]) is a doubly ranked set $M=\left(M_{m, n}\right)$ equipped with two operations denoted by ${ }^{\circ}$ (circle) and D (box):

$$
\text { 。: } M_{m, n} \times M_{n, k} \rightarrow M_{m, k}, \quad \text { D : } M_{m, n} \times M_{m^{\prime}, n^{\prime}} \rightarrow M_{m+m^{\prime}, n+n^{\prime}}
$$

for all $\left.m, n, k, m^{!}, n^{!}\right) 0$, which are associative in the obvious way and satisfy the distributivity law $\left(f^{\circ} g\right) \mathrm{D}\left(f^{\prime} \circ g^{\prime}\right)=\left(f \mathrm{D} f^{\prime}\right) \circ\left(g \mathrm{D} g^{\prime}\right)$ whenever all the above operations are defined. Moreover, it is equipped with a sequence of constants $\left.e_{n} \in M_{n, n}(n) 0\right)$, called units, such that

$$
e_{m} \circ f=f=f \circ e_{n}, \quad e_{0} D f=f=f D e_{0}
$$

for all $f \in M_{m, n}$ and all $\left.m, n\right) 0$, and the additional condition $e_{m} D e_{n}=e_{m+n}$ holds true for all $m, n) 0$. Notice that, due to the last equation, the elements $e_{n}$ $(n \geq 2)$ are uniquely determined by $e_{1}$. From now on $e_{1}$ will be simply denoted by $e$. Submagmoids, morphisms, congruences and quotients of magmoids are defined in the obvious way.

An elegant characterization of a congruence can be achieved by means of the notion of the context. In a magmoid $M$ an $(m, n)$-context is a 4-tuple $\omega=$ $\left(g_{1}, f_{1}, f_{2}, g_{2}\right)$, with $f_{i} \in M_{m_{i}, n_{i}}(i=1,2), g_{1} \in M_{a, m_{1}+m+m_{2}}, g_{2} \in M_{n_{1}+n_{+} n_{2}, b}$, where $a, b \in \mathrm{N}$.

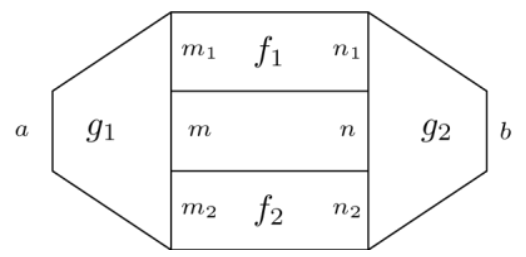

The set of all $(m, n)$-contexts is denoted $\operatorname{Cont}_{m, n}(M)$. For any $f \in M_{m, n}$ and $\omega=\left(g_{1}, f_{1}, f_{2}, g_{2}\right)$ as above, we write $\omega[f]=g_{1} \circ\left(f_{1} \mathrm{D} f \mathrm{D} f_{2}\right) \circ g_{2}$; note that $\omega[f] \in M_{a, b}$.

Proposition 1 (cf. [11]). The equivalence $\sim=\left(\sim_{m, n}\right)$ on the magmoid $M=$ $\left(M_{m, n}\right)$ is a congruence whenever, for all $\left.m, n\right) 0, f, g \in M_{m, n}$ and all $\omega \in$ $\operatorname{Cont}_{m, n}(M)$

$$
f \sim_{m, n} g \text { implies } \omega[f] \sim_{a, b} \omega[g] .
$$

We say that a doubly ranked family $L=\left(L_{m, n}\right)$ is a subset of the magmoid $M=\left(M_{m, n}\right)$ (notation $L \subseteq M$ ), whenever $L_{m, n} \subseteq M_{m, n}$ for all $m, n \in \mathrm{N}$. The boolean and the magmoid operations on subsets of $M$ are defined in the obvious way.

Two elements of a magmoid $M$ are equivalent modulo the syntactic congruence of a subset $L \subseteq M$, whenever they have the same set of contexts with respect to $L$. Formally, let $L$ be a subset of the magmoid $M$ and $f \in M_{m, n}$, we set $C_{L}(f)=\left\{\omega \mid \omega \in\right.$ Cont $\left._{m, n}(M), \omega[f] \in L\right\}$.

Proposition 2 (cf. [11]). The equivalence $\sim_{L}$ on $M$ defined by 


$$
f \sim_{L, m, n} g, \text { whenever } C_{L}(f)=C_{L}(g)
$$

is a congruence.

Given a magmoid $M$ and a set $L \subseteq M, \sim_{L}$ is called the syntactic congruence of $L$ and the quotient magmoid $M_{L}=M / \sim_{L}$ is the syntactic magmoid of $L$. Thus, for all $m, n) 0$, the set $\left(M_{L}\right)_{m, n}$ can be identified with the set consisting of all distinct contexts of the elements of $M_{m, n}$, i.e., we may write $\left(M_{L}\right)_{m, n}=\left\{C_{L}(f) \mid f \in M_{m, n}\right\}$ whereas, the operations of $M_{L}$ are given by the formulas:

$$
C_{L}(f) \circ C_{L}(g)=C_{L}(f \circ g), \quad C_{L}(f) D C_{L}(g)=C_{L}(f \mathrm{D} g) .
$$

The syntactic magmoid is characterized by the following universal property: for any magmoid epimorphism $h: M \rightarrow N$, such that $h^{-1}(h(L))=L$, there exists a unique magmoid morphism $\bar{h}: N \rightarrow M_{L}$ such that $\bar{h} \circ h=h_{L}$, where $h_{L}: M \rightarrow M_{L}$ is the canonical projection onto the quotient. Thus $M_{L}$ is unique up to isomorphism.

\section{Recognizability of Fuzzy Graphs}

Fuzzy graphs can be organized into a magmoid by virtue of two operations: product or composition corresponding to ${ }^{\circ}$ and sum corresponding to $D$. Let $F$ be an $(m, n)$-graph and $G$ is an $(n, k)$-graph represented respectively by

$$
\left.\left(V_{\kappa}, E_{\lambda}, s, t, l, \text { begin, end }\right) \text { and }\left(V_{K^{\prime}}^{!}, E_{\lambda^{\prime}}^{!}, s^{!}, t^{!}, l^{\prime} \text {, begin!, end }\right)^{\prime}\right) \text {, }
$$

then their product $F \circ G$ is the $(m, k)$-graph

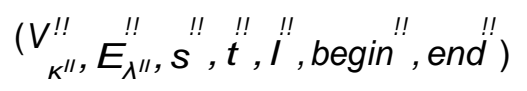

obtained by taking the disjoint union of $F$ and $G$ and then identifying the $t^{\text {th }}$ end node $v$ of $F$ with the $i^{\text {th }}$ begin node $v^{\prime}$ of $G$, for all $i \in\{1, \ldots, n\}$; for the resulting node $v^{! !}$we set $\kappa^{! !}\left(v^{! !}\right)=\max \left\{\kappa(v), \kappa\left(v^{\prime}\right)\right\}$. Additionally, begin!" $=$ begin and end!" = end! . The sum $F D G$ of arbitrary graphs $F$ and $G$ is their disjoint union with their sequences of begin nodes concatenated and similarly for their end nodes.

For instance let $\Sigma=\{a, b, c\}$, with $\operatorname{rank}(a)=(2,1), \operatorname{rank}(b)=(1,1)$ and $\operatorname{rank}(c)=(1,2)$. In the following pictures, edges are represented by boxes, nodes by dots, and the sources and targets of an edge by directed lines that enter and leave the corresponding box, respectively. The order of the sources and targets of an edge is the vertical order of the directed lines as drawn in the pictures. The membership grades are omitted for simplicity. We display two graphs $F \in F G R_{3,4}(\Sigma)$ and $G \in F G R_{4,2}(\Sigma)$, where the th begin node is indicated by $b_{i}$, and the ith end node by $e_{i}$. 

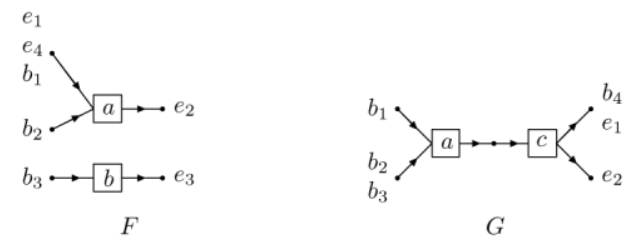

Then their product $F \circ G$ and their sum $F D G$ are respectively the $(3,2)$ and the $(7,6)$-graphs
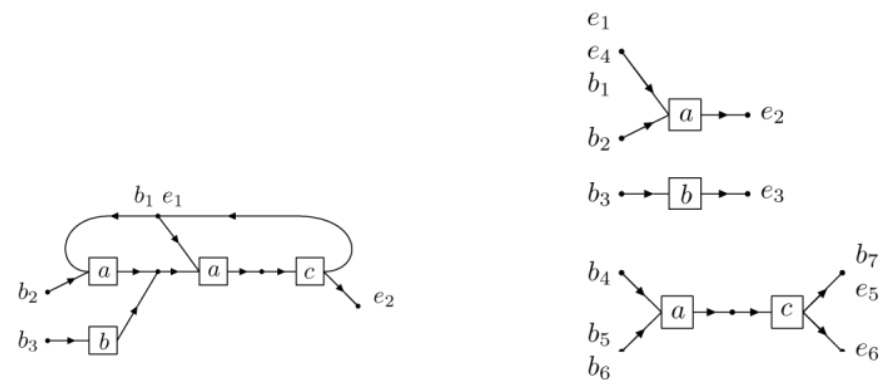

For every $n \in \mathrm{N}$ we denote by $E_{n}$ the discrete $(n, n)$-graph with nodes $x_{1}, \ldots, x_{n}$, begin and end sequence $x_{1} \cdots x_{n}$ and $k\left(x_{i}\right)=0$ for all $i$; we write $E$ for $E_{1}$. It can be verified that $F G R(\Sigma)=\left(F G R_{m, n}(\Sigma)\right)$ with the operations defined above is a magmoid, whose units are the graphs $E_{n}, n \geq 0$.

Recognizability inside magmoids can be defined in a way similar to that of monoids. A congruence $\sim=\left(\sim_{m, n}\right)$ on a magmoid $M=\left(M_{m, n}\right)$ saturates $L \subseteq M$ whenever, for all $m, n \geq 0$, the subset $L_{m, n}$ is a union of $\sim_{m, n}$-classes. If, for all $m, n \geq 0$, the congruence $\sim_{m, n}$ has finite index (i.e., finite number of equivalence classes) we say that $\sim$ has locally finite index. Moreover, a magmoid $M=\left(M_{m, n}\right)$ is said to be locally finite if, for all $m, n \geq 0$, the set $M_{m, n}$ is finite.

Definition 1. A subset $L$ of $F G R(\Sigma)$ is called (syntactic) recognizable if there exists a locally finite magmoid $N=\left(N_{m, n}\right)$ and a morphism $h: F G R(\Sigma) \rightarrow N$, so that $L=h^{-1}(P)$, for some $P \subseteq N$.

We denote by $\operatorname{Rec}(F G R(\Sigma))$ the class of all syntactically recognizable subsets of $F G R(\Sigma)$. The elements of $\operatorname{Rec}(F G R(\Sigma))$ are called recognizable fuzzy graph languages. From the described fuzzy recognizability notion and from the construction of the syntactic magmoid, similarly with crisp recognizability, we deduce

Theorem 1. Let $L \subseteq F G R(\Sigma)$, the following conditions are equivalent:

1. Lis recognizable;

2. $L$ is saturated by a congruence of a locally finite index;

3. $\sim_{L}$ has locally finite index; 
4. $\operatorname{card}\left\{C_{L}(F) \mid F \in F G R_{m, n}(\Sigma)\right\}$ is finite for all $m, n \in \mathrm{N}$;

5. the syntactic magmoid $F G R(\Sigma)_{L}$ is locally finite.

Corollary 1. The class $\operatorname{Rec}(F G R(\Sigma))$ of all recognizable fuzzy graph languages is closed under finite union, intersection, complement and inverse morphisms of magmoids.

Proof. Combine the above theorem with Proposition 3 of [11].

Proposition 3. Let $\Sigma$ be a doubly ranked alphabet and $a, b \in \Sigma, a j=b$, the fuzzy graph language $L^{a, b} \subseteq F G R(\Sigma)$ consisting of all graphs that have an equal

number of labels $a$ and $b$ on edges with memebeship grade greater or equal to $k$, $k \in[0,1]$, is not recognizable

Proof. For every $G \in F G R(\Sigma)$ and every $\omega=\left(G_{1}, F_{1}, F_{2}, G_{2}\right)$ we denote by $|G|_{a}$ the number of $a$ 's occurring as labels of edges with membership grade greater or equal to $k$ in $G$ and

$$
|\omega|_{a}=\left|G_{1}\right|_{a}+\left|F_{1}\right|_{a}+\left|F_{2}\right|_{a}+\left|F_{2}\right|_{a} .
$$

Let $G \in F G R(\Sigma)$, we observe that for every $\omega, \omega^{!} \in \underset{k}{C_{k}^{a, b}}(G)$ it holds

$$
|\omega|_{a}-|\omega|_{b}=\left|\omega^{\prime}\right|_{a}-\left|\omega^{\prime}\right|_{b}=|G|_{b}-|G|_{a}
$$

We can easily verify that the function

$$
G \stackrel{\varphi_{m, n}}{\rightarrow}|G|_{b}-|G|_{a}, \quad G \in G R_{m, n}(\Sigma)
$$

is a bijection of the set $\left(F G R(\Sigma)_{L_{k}^{a, b}}\right)_{m, n}$ on the set of integers $Z$. Furthermore it holds

$$
\varphi\left(G \circ G^{\prime}\right)=\varphi(G)+\varphi\left(G^{\prime}\right), \varphi\left(G D G^{\prime}\right)=\varphi(G)+\varphi\left(G^{\prime}\right) \text { and } \varphi\left(E_{n}\right)=0_{n, n}
$$

and thus, the syntactic magmoid of $L_{k}^{a, b}$ is isomorphic to the magmoid associated with the commutative monoid of the additive integers. Since this is locally infinite from Theorem 1 we derive that this language is not recognizable.

Remark 1. Note that $L_{0}^{a, b}$ consists of all fuzzy graphs with an equal number of a's and b's. As we have shown in [11], the syntactic magmoid of the crisp graph language that consists of all graphs with an equal number of $a$ 's and b's in their labels, is also isomorphic with the same magmoid and in this respect the present result constitutes a generalization for fuzzy graph languages.

Proposition 4. The fuzzy graph language $L_{1} \subseteq F G R(\Sigma)$ consisting of all graphs that have exactly $k$ edges $(k \geq 1)$ with membership grade 1 is recognizable.

Proof. For every $G \in F G R(\Sigma)$ let $|G|_{1}$ be the number of edges of $G$ with membership grade 1 and for every $\omega=\left(G_{1}, F_{1}, F_{2}, G_{2}\right)$ we set 


$$
|\omega|_{1}=\left|G_{1}\right|_{1}+\left|F_{1}\right|_{1}+\left|F_{2}\right|_{1}+\left|F_{2}\right|_{1}
$$

It holds:

- $|G|_{1}=0$, whenever for every $\omega \in C_{L_{1}}(G),|\omega|_{1}=k$,

- $|G|_{1}=1$, whenever for every $\omega \in C_{L_{1}}(G),|\omega|_{1}=k-1$,

- $|G|_{1}=k-1$, whenever for every $\omega \in C_{L_{1}}(G),|\omega|_{1}=1$,

- $|G|_{1}=k$, whenever for every $\omega \in C_{L_{1}}(G),|\omega|_{1}=0$,

- $|G|_{1} \geq k+1$, whenever $C_{L_{1}}(G)=\varnothing$.

The function $\varphi_{m, n}:\left(F G R(\Sigma)_{L_{1}}\right)_{m, n} \rightarrow\{0,1, \ldots, k, \alpha\}$, sending the syntactic class of every graph $G \in F G R(\Sigma)_{m, n}$ to $0,1, \ldots, k$ or $\alpha$, whenever $|G|_{1}=0,1, \ldots, k$ or ) $k+1$ respectively, is a bijection.

Now let $M(A)=\left(M(A)_{m, n}\right)$ be the magmoid associated with the commutative monoid $A=\{0,1, \ldots, k, \alpha\}$ whose operation is given by the following table.

\begin{tabular}{c|ccccc}
+ & 0 & 1 & $\ldots$ & $\mathrm{k}$ & $\alpha$ \\
\hline 0 & 0 & 1 & $\ldots$ & $\mathrm{k}$ & $\alpha$ \\
1 & 1 & 2 & $\ldots$ & $\alpha$ & $\alpha$ \\
$:$ & $:$ & $:$ &.. & $:$ & $:$ \\
$\mathrm{k}$ & $\mathrm{k}$ & $\alpha$ & $\ldots$ & $\alpha$ & $\alpha$ \\
$\alpha$ & $\alpha$ & $\alpha$ & $\ldots$ & $\alpha$ & $\alpha$
\end{tabular}

It holds:

$$
\varphi\left(G \circ G^{\prime}\right)=\varphi(G)+\varphi\left(G^{\prime}\right), \varphi\left(G D G^{\prime}\right)=\varphi(G)+\varphi\left(G^{\prime}\right) \text { and } \varphi\left(E_{n}\right)=0_{n, n}
$$

and hence the syntactic magmoid of $L_{1}$ is isomorphic to $M(A)$. This is a locally finite magmoid and hence from Theorem 1 we deduce that $L_{1} \in \operatorname{Rec}(F G R(\Sigma))$.

Proposition 5. Given a finite doubly ranked set $\Sigma$, the class $\operatorname{Rec}(F G R(\Sigma))$ is closed under D-operation.

Proof. Similar with the corresponding proof for crisp graph languages (see [11]).

Proposition 6. The fuzzy graph language $L_{d p} \subseteq F G R_{1,1}(\Sigma)$ that consists of all graphs that have at least one directed path from the begin node to the end node through edges with membership grade 1 is recognizable.

Proof. We define the following equivalence on $F G R_{m, n}(\Sigma): G_{1} \sim_{p} G_{2}$ whenever the next two items are equivalent there exists a directed path from the $i^{\text {th }}$ begin node of $G_{1}$ to the $j^{\text {th }}$ end node of $G_{1}$ through edges with membership grade 1 
if and only if there exists a directed path from the $i^{\text {th }}$ begin node of $G_{2}$ to the $j^{\text {th }}$ end node of $G_{2}$ through edges with membership grade 1. It holds:

$$
G_{1} \sim_{p} G_{2} \text { and } G_{1}^{!} \sim_{p} G_{2}^{\prime} \text { implies } G_{1} D G_{1}^{\prime} \sim_{p} G_{2} D G_{2}^{!}
$$

and similarly for $\circ$, hence $\sim_{p}$ is a congruence which trivially saturates $L_{d p}$ and thus by Theorem 1 we get that $L_{d p}$ is recognizable.

Proposition 7. The fuzzy graph language $L_{s c}$ of all strongly connected graphs is recognizable.

Proof. Let $G=\left(V_{k}, E_{\lambda}, s, t\right.$, l, begin, end $) \in F G R_{m, n}(\Sigma)$. If there exist nodes $u, v \in V$ without a directed path from $u$ to $v$ or backward and are not both either in the begin or the end sequence of $G$, then $C_{L_{s c}}(G)=\varnothing$.

Assume now that every node of $G$ that doesn't appear in the begin and the end sequences of $G$ has directed paths back and forth with every other node of $G$. Then there exist contexts of $G$ with respect to $L_{s c}$. The possible different such contexts correspond to the different ways we can arrange the $m+n$ begin and end marks to the nodes of $G$ in a way that each node that requires at least one directed path connecting it with another node appears at least once in the begin or the end sequence of $G$. This is clearly a finite number, hence

$$
\operatorname{card}\left\{C_{L_{s c}}(G) \mid G \in F G R_{m, n}(\Sigma)\right\}<\infty
$$

and by Theorem 1 the language $L_{s c}$ is recognizable.

Fuzzy graph language recognizability can also be characterized through left derivatives in a result that is a generalization of the fundamental fact that a string language is recognizable, if and only if, it has finitely many left derivatives, if and only if, it has finitely many right derivatives [18]. Let $L \subseteq F G R(\Sigma)$ and $\omega \in \operatorname{Cont}_{m, n}(F G R(\Sigma))$. The left derivative of $L$ at $\omega$ is defined as

$$
\omega^{-1} L=\left\{G \in F G R_{m, n}(\Sigma) \mid \omega[G] \in L\right\} .
$$

Proposition 8. The fuzzy graph language $L \subseteq F G R(\Sigma)$ is recognizable, if and only if, $\operatorname{card}\left\{\omega^{-1} L \mid \omega \in\right.$ Cont $\left._{m, n}(F G R(\Sigma))\right\}<\infty$, for all $m, n \in \mathrm{N}$.

Proof. As in the case of crisp graph languages (see Proposition 5 of [11]).

By virtue of this proposition we prove the following result.

Proposition 9. Let $L \subseteq F G R(\Sigma)$ be the binary fuzzy graph language that consists only of graphs containing only nodes with membership grade 1 . Then $L$ is recognizable if and only if $L^{f c}$ is recognizable.

Proof. Assume that $L \in \operatorname{Rec}(F G R(\Sigma))$ and $m, n \in \mathrm{N}$, then by the previous proposition

$\operatorname{card}\left\{\omega^{-1} L \mid \omega \in \operatorname{Cont}_{m, n}(F G R(\Sigma))\right\}<\infty$.

Let $\omega^{-1} L, \ldots, \omega^{-1} L$ be representatives of the distinct left derivatives of $L$. We shall prove that ${ }^{k}\left(\omega_{1}^{f c}\right)^{-1} L, \ldots,\left(\omega_{k}^{f c}\right)^{-1} L$ are all the distinct left derivatives of $L^{f c}$. For every $\omega=\left(G_{1}, F_{1}, F_{2}, G_{2}\right)$, we set 


$$
\omega^{f c}=\left(G_{1}^{f c}, F_{1}^{f c}, F_{2}^{f c}, G_{2}^{f c}\right) .
$$

Note that for any graph $G \in F G R(\Sigma)$ it holds $\left(G^{f c}\right)^{f c}=G$. Now, let $\omega \in$ Cont $_{m, n}(F G R(\Sigma))$, then for any graph $G \in F G R(\Sigma)$ it holds

$$
\omega[G] \in L^{f c} \Leftrightarrow(\omega[G])^{f c} \in L \stackrel{*}{\Leftrightarrow} \omega^{f c}\left[G^{f c}\right] \in L .
$$

Since we assumed that $\omega_{1}^{-1} L, \ldots, \omega_{k}^{-1} L$ are all the distinct left derivatives of $L$, from the last we deduce that there exists $1 \leq i \leq k$ such that

$$
\omega_{i}\left[G^{f c}\right] \in L \Leftrightarrow\left(\omega_{i}\left[G^{f c}\right]\right)^{f c} \in L^{f c} \stackrel{*}{\bullet} \omega_{i}^{f c}[G] \in L^{f c} .
$$

Hence the context $\omega$ is identified with one of $\omega_{1}^{f c}, \ldots, \omega_{k}^{f c}$, and thus $L^{f c}$ has finite distinct left derivatives which by Proposition 8 concludes the proof. Notice that in the equivalences $\stackrel{*}{\square}$ we used the equality $(\omega[G])^{f c}=\omega^{f c}\left[G^{f c}\right]$ which holds only in the case that the graph $G$ has only nodes with membership grade 1 .

\section{Conclusion}

Fuzzy graphs were defined as an extension of the notion given by Rosenfeld in [32], i.e., directed (hyper)graphs with fuzzy sets of nodes and fuzzy sets of edges. It turns out that the set of all fuzzy graphs can be structured into a magmoid with operations product (graph composition) and sum (disjoint union). By virtue of this algebraic structure we introduced a syntactic recognizability notion for sets of fuzzy graphs employing the syntactic magmoid in a role analogous to the syntactic monoid of string languages. This approach shall allow us to explore the fuzzy case for existing crisp graph theoretic methods and techniques involving formal verification $[3,4,13]$ and natural language processing [31] as well as for the syntactic complexity of string and graph languages [14, 15, 22].

\section{References}

[1] Arnold, A., Dauchet, M.: Théorie des magmoides. I. RAIRO Inform. Théor. 12, 235-257 (1978)

[2] Arnold, A., Dauchet, M.: Théorie des magmoides. II. RAIRO Inform. Théor. 13, 135-154 (1979)

[3] Blume, C.: Recognizable Graph Languages for the Verification of Dynamic Systems. LNCS 6372, 384-387 (2010)

[4] Blume, C., Bruggink, H. J. S., Friedrich, M., König, B.: Treewidth, pathwidth and cospan decompositions with applications to graphaccepting tree automata. Journal of Visual Languages \& Computing http://dx.doi.org/10.1016/j.jvlc.2012.10.002 
[5] Bozapalidis, S., Bozapalidou, O. L.: On the recognizability of fuzzy languages I. Fuzzy Sets and Systems 157, 2394-2402 (2006)

[6] Bozapalidis, S., Bozapalidou, O. L.: On the recognizability of fuzzy languages II. Fuzzy Sets and Systems 159, 107-113 (2008)

[7] Bozapalidis, S., Bozapalidou, O. L.: Fuzzy tree language recognizability. Fuzzy Sets and Systems 161, 716-734 (2010)

[8] Bozapalidis, S., Kalampakas, A.: A Finite Complete Set of Equations Generating Graphs. Discrete Mathematics and Theoretical Computer Science, LNCS 2731, 118-128 (2003)

[9] Bozapalidis, S., Kalampakas, A.: An Axiomatization of Graphs. Acta Inform. 41, 19-61 (2004)

[10] Bozapalidis, S., Kalampakas, A.: Automata on patterns and graphs. Proceedings of the 1st International Conference on Algebraic Informatics, 3152, Aristotle Univ. of Thessaloniki (2005)

[11] Bozapalidis, S., Kalampakas, A.: Recognizability of graph and pattern languages. Acta Inform. 42, 553-581 (2006)

[12] Bozapalidis, S., Kalampakas, A.: Graph Automata. Theoret. Comput. Sci. 393, 147-165 (2008)

[13] Bruggink, H. J. S., König, B.: A logic on subobjects and recognizability. IFIP-AICT 323, 197-212 (2010)

[14] Brzozowski, J., Ye, Y.: Syntactic Complexity of Ideal and Closed Languages. LNCS 6795, 117-128 (2011)

[15] Brzozowski, J., Li, B., Ye, Y.: Syntactic complexity of prefix-, suffix-, bifix-, and factor-free regular languages. Theoret. Comput. Sci. 449, 37-53 (2012)

[16] Courcelle, B.: The Monadic Second-Order Logic of Graphs. I. Recognizable Sets of Finite Graphs. Inform. and Comput. 85, $12-75$ (1990)

[17] Courcelle, B., Weil, P.,: The recognizability of sets of graphs is a robust property. Theor. Comput. Sci. 342, 173-228 (2005)

[18] Eilenberg, S.: Automata, Languages and Machines, vol. A, Academic Press (1974)

[19] Engelfriet, J., Vereijken, J. J.: Context-free graph grammars and concatenation of graphs. Acta Inform. 34, 773-803 (1997)

[20] Ésik, Z., Liu, G.: Fuzzy tree automata. Fuzzy Sets and Systems 158, 1450$1460(2007)$

[21] Gécseg, F., Steinby, M.: Tree Automata, Akademiai Kiado, Budapest (1984) 
[22] Kalampakas, A.: The Syntactic Complexity of Eulerian Graphs. Proceedings of the 2nd International Conference on Algebraic Informatics, LNCS 4728, 208-217 (2007)

[23] Kalampakas, A.: Graph Automata: The Algebraic Properties of Abelian Relational Graphoids. LNCS 7020, 168-182 (2011)

[24] Konstandinidis, S., Sântean, N., Yu, S.: Fuzzification of rational and recognizable sets. Fundamenta Informaticae 76, 413-447 (2007)

[25] Kuich, W., Rahonis, G.: Fuzzy regular languages over finite and infinite words. Fuzzy Sets and Systems 157, 1532-1549 (2006)

[26] Louskou-Bozapalidou, O.: Non-deterministic recognizability of fuzzy languages. Applied Mathematical Sciences 1, 821-826 (2007)

[27] Malik, D. S., Mordeson, J. N., Sen, M. K.: Minimization of fuzzy finite automata. Information Sciences 113, 323-330 (1999)

[28] Mordeson, J. N., Nair, P. S.: Fuzzy Graphs and Fuzzy Hypergraphs. Physica-Verlag, Heidelberg (2000)

[29] Mordeson, J. N., Malik, D.: Fuzzy Automata and Languages: Theory and Applications. Chapman \& Hall/CRC, London (2002)

[30] Petković, T., Varietes of fuzzy languages. In Proc. 1st International Conference on Algebraic Informatics, 197-205, Aristotle University of Thessaloniki (2005)

[31] Quernheim, D., Knight, K.: Towards Probabilistic Acceptors and Transducers for Feature Structures. In Proc. 6th Workshop Syntax, Semantics and Structure in Statistical Translation, 76-85, Association for Computational Linguistics (2012)

[32] Rosenfeld, A.: Fuzzy graphs. In: L.A. Zadeh, K.S. Fu, K. Tanaka, M. Shimura (Eds.), Fuzzy Sets and Their Applications to Cognitive and Decision Processes, 77-95, Academic Press, New York (1975)

[33] Rahonis, G.: Infinite fuzzy computations. Fuzzy Sets and Systems 153, 275-288 (2005)

[34] Rahonis, G.: Fuzzy Languages. Handbook of Weighted Automata, Monographs in Theoretical Computer Science, 481-517 (2009)

[35] Zadeh, L. A.: Fuzzy sets. Information and Control 8, 338-353 (1965) 